\title{
EL TEOREMA DE DULAC
}

\section{Renato Benazic ${ }^{1}$, Claudio Espinoza ${ }^{2}$ \& Liliana Jurado ${ }^{3}$}

Resumen: En el presente trabajo se demuestra el Teorema de Dulac el cual establece que si un campo de dimensión compleja dos, cuya parte lineal tiene dos autovalores resonantes en el dominio Poincaré entonces él es analíticamente localmente conjugado a un campo polinomial. Se estudia también el comportamiento geométrico de sus órbitas en la vecindad de la singularidad.

Palabras clave: Ecuaciones Diferenciales Complejas, Singularidades Aisladas, Dinámica compleja.

\section{DULAC'S THEOREM}

\begin{abstract}
In this work is proven the Dulac's Theorem, which establishes that if a complex 2-dimension vector field, which linear part has two resonant eigenvectors in the Poincare domain then it is locally analytically conjugate to a polinomial vector field. Is is also studied the geometric behavior of its orbits in a neighborhood of a singularity.

Key words: Complex Differential Equations, Isolated Singularities, Complex Dynamics.
\end{abstract}

\section{Introducción}

Sea $U \subseteq \mathbb{C}^{n}$ un abierto, un campo vectorial $Z=\left(Z_{1}, \ldots, Z_{n}\right): U \rightarrow \mathbb{C}^{n}$ es llamado holomorfo si y sólo si todas sus funciones coordenadas $Z_{1}, \ldots, Z_{n}: U \rightarrow \mathbb{C}$ son funciones holomorfas. Denotaremos por $\mathcal{X}(U)$ al conjunto de todos los campos vectoriales definidos en el abierto $U \subseteq \mathbb{C}^{n}$.

Un punto $p \in U$ es llamado singularidad de $Z \in \mathcal{X}(U)$ si y sólo si $Z(p)=0$. Caso contrario $p$ es llamado punto regular de $Z$. Denotaremos por $\operatorname{Sing}(Z)$ al conjunto de todos los puntos singulares del campo $Z$.

Dados $Z \in \mathcal{X}(U), z_{0} \in U$ y $T_{0} \in \mathbb{C}$, su Problema de Valor Inicial (P.V.I.) o Problema de Cauchy asociado es dado por:

$$
\mid \begin{array}{ll}
z^{\prime} & =Z(z) \\
z\left(T_{0}\right) & =z_{0}
\end{array}
$$

Una solución de (1) es una función holomorfa $\varphi: D \rightarrow U$ donde $D \subseteq \mathbb{C}$ es un disco abierto, tal que

1. $T_{0} \in D$.

2. $\varphi^{\prime}(T)=Z(\varphi(T)) ; \forall T \in D$.

\footnotetext{
${ }^{1}$ Universidad Nacional Mayor de San Marcos - Facultad de Ciencias Matemáticas, Instituto de Matemática y Ciencias Afines - IMCA

${ }^{2}$ Universidad Nacional Mayor de San Marcos - Facultad de Ciencias Matemáticas

${ }^{3}$ Universidad Nacional Mayor de San Marcos - Facultad de Ciencias Matemáticas
} 
3. $\varphi\left(T_{0}\right)=z_{0}$.

El Teorema de existencia y unicidad (ver [4]) asegura que el PVI (1) admite una única solución $\varphi_{\left(T_{0}, z_{0}\right)}$, la cual está definida en un disco cerrado $D_{\alpha}\left[T_{0}\right]$ de radio suficientemente pequeño $\alpha>0$, centrado en $T_{0}$. Más aún, sea $\Delta\left[z_{0} ; r\right]$ un polidisco cerrado contenido en $U$, si $Z \in \mathcal{X}(U)$ es Lipschitz en $\Delta\left[z_{0} ; r\right]$ entonces se puede demostrar que (ver [1]) existe poliradio $r^{\prime}<r$ y existe $0<\alpha^{\prime}<\alpha$ tales que para todo $z \in \Delta\left[z_{0} ; r^{\prime}\right]$ existe una única solución $\varphi_{z}: D_{\alpha^{\prime}}\left[T_{0}\right] \rightarrow \Delta\left[z_{0} ; r\right]$ del PVI:

$$
\mid \begin{aligned}
& w^{\prime}=Z(w) \\
& w\left(T_{0}\right)=z
\end{aligned}
$$

Como consecuencia de este último resultado, podemos definir la función

$$
\varphi_{Z}: D_{\alpha^{\prime}}\left[T_{0}\right] \times \Delta\left[z_{0} ; r^{\prime}\right] \rightarrow \Delta\left[z_{0} ; r\right]
$$

mediante

$$
\varphi_{Z}(T, z)=\varphi_{z}(T)
$$

Esta función $\varphi$ es llamada Flujo Local asociado a $Z$ alrededor de $\left(T_{0}, z_{0}\right)$. Es claro que el flujo satisface las siguientes condiciones:

1. $\frac{\partial \varphi}{\partial T}(T, z)=Z(\varphi(T, z)), \forall(T, z) \in D_{\alpha^{\prime}}\left(T_{0}\right) \times \Delta\left(z_{0} ; r^{\prime}\right)$.
2. $\varphi\left(T_{0}, z\right)=z, \forall z \in \Delta\left(z_{0} ; r^{\prime}\right)$.

3. $\varphi$ es una función holomorfa.

Para simplificar, en lo sucesivo, trabajaremos siempre con el valor inicial $T_{0}=0$.

Definición 1.1. Sean $U_{1}, U_{2} \subseteq \mathbb{C}^{n}$ abiertos, $Z_{1} \in \mathcal{X}\left(U_{1}\right), Z_{2} \in \mathcal{X}\left(U_{2}\right), p_{1} \in U_{1}, p_{2} \in U_{2} y$ consideremos sus flujos locales asociados

$$
\varphi_{1}: D_{\delta}(0) \times \Delta\left(p_{1} ; r^{\prime}\right) \rightarrow \Delta\left(p_{1} ; r\right) \quad \varphi_{2}: D_{\delta}(0) \times \Delta\left(p_{2} ; r^{\prime}\right) \rightarrow \Delta\left(p_{2} ; r\right)
$$

Decimos que $Z_{1}$ es localmente topológicamente (resp. analíticamente) conjugado a $Z_{2}$ alrededor de $p_{1}$ y $p_{2}$, lo que denotamos $Z_{1} \sim_{\text {top }} Z_{2}$ (resp. $Z_{1} \sim_{\text {ana }} Z_{2}$ ) si y sólo si existen vecindades abiertas $V_{1} \subseteq U_{1}, V_{2} \subseteq U_{2}$ de $p_{1}$ y $p_{2}$ respectivamente, y existe $h: V_{1} \rightarrow V_{2}$ homeomorfismo (resp. biholomorfismo) llamado conjugación topológica local (resp. conjugación analítica local) tal que

$$
h\left(\varphi_{1}(T, z)\right)=\varphi_{2}(T, h(z)), \quad \forall(T, z) \in D_{\delta}(0) \times V_{1}
$$

El hecho de que dos campos sean analíticamente localmente conjugados, significa geométricamente que sus soluciones son indistinguibles salvo biholomorfismos.

El siguiente resultado, cuya demostración puede ser encontrada en ([1]), nos proporciona un criterio bastante útil para determinar si dos campos son conjugados, sin necesidad de usar los flujos.

Proposición 1.1. Sean $U_{1}, V_{1}, U_{2}, V_{2} \subseteq \mathbb{C}^{n}$ abiertos, $Z_{1} \in \mathcal{X}\left(U_{1}\right), Z_{2} \in \mathcal{X}\left(U_{2}\right), p_{1} \in V_{1} \subseteq U_{1}$, $p_{2} \in V_{1} \subseteq U_{2}$ y $h: V_{1} \rightarrow V_{2}$ un biholomorfismo. Se cumple $h$ es una conjugación analítica local entre $Z_{1}$ y $Z_{2}$ alrededor de $p_{1}$ y $p_{2}$ si y sólo si

$$
h^{\prime}(z) \cdot Z_{1}(z)=Z_{2}(h(z)), \forall z \in V_{1}
$$

En la vecindad de un punto regular, las soluciones de $Z$ pueden ser rectificadas, más específicamente, tenemos el siguiente resultado. 
Teorema 1.1. (Teorema del Flujo Tubular) Sean $U \subseteq \mathbb{C}^{n}$ un abierto y $Z \in \mathcal{X}(U)$. Si $z_{0} \in U$ es un punto regular de $Z$ entonces $Z$ es localmente analíticamente conjugado al campo constante $Y=(1,0, \ldots, 0)$ alrededor de $z_{0}$ y 0 .

Para demostrar este resultado, el lector puede referirse a ([6]) y adaptarlo fácilmente al caso complejo.

En virtud del Teorema del Flujo Tubular, podemos considerar satisfactorio el conocimiento geométrico local de las soluciones de una ecuación diferencial alrededor de un punto regular. Resta entender cómo es este comportamiento en la vecindad de una singularidad.

\section{Campos vectoriales holomorfos con parte lineal no nula}

En lo sucesivo, nos restringiremos a la dimensión compleja 2.

Definición 2.1. Sea $U \subseteq \mathbb{C}^{2}$ abierto y $Z \in \mathcal{X}(U)$. Decimos que $z_{0} \in U$ es una singularidad aislada de $Z$ si y sólo si $z_{0} \in \operatorname{Sing}(Z)$ y existe $V \subseteq U$ vecindad abierta de $z_{0}$ tal que $\operatorname{Sing}(Z) \cap\left(V-\left\{z_{0}\right\}\right)=\emptyset$.

Sea $U \subseteq \mathbb{C}^{2}$ abierto, $Z \in \mathcal{X}(U)$ y $0 \in U$ singularidad aislada de $\dot{Z}$, luego existe $V \subseteq U$ vecindad abierta de 0 tal que

$$
Z(z)=\left(\sum_{|Q| \geq 1} a_{1, Q} z^{Q}, \sum_{|Q| \geq 1} a_{2, Q} z^{Q}\right) \forall z \in V
$$

y

$$
Z(z) \neq(0,0), \forall z \in V-\{0\}
$$

Sabemos que su derivada $D Z(0)$ es una transformación lineal de $\mathbb{C}^{2}$ en $\mathbb{C}^{2}$ y que su matriz asociada (en la base canónica de $\mathbb{C}^{2}$ ) es dada por

$$
\left[\begin{array}{ll}
a_{1,(1,0)} & a_{1,(0,1)} \\
a_{2,(1,0)} & a_{2,(0,1)}
\end{array}\right] \in \mathbb{C}^{2 \times 2}
$$

la cual también la denotaremos por $D Z(0)$. $D Z(0)$ es llamada la parte lineal de $Z$ en $0 \in \mathbb{C}^{2}$ y diremos que $Z$ tiene parte lineal no nula en $0 \in \mathbb{C}^{2}$ si y sólo si $D Z(0) \neq 0$. Si $D Z(0) \neq 0$, por el Teorema de la Forma Canónica de Jordan basta considerar los casos:

$$
D Z(0)=\left[\begin{array}{cc}
\lambda_{1} & 0 \\
0 & \lambda_{2}
\end{array}\right], \quad D Z(0)=\left[\begin{array}{cc}
\lambda & 0 \\
0 & \lambda
\end{array}\right] \quad \text { ó } \quad D Z(0)=\left[\begin{array}{cc}
\lambda & 1 \\
0 & \lambda
\end{array}\right]
$$

Desde que $G L\left(\mathbb{C}^{2}\right)$ es abierto y denso en $\mathbb{C}^{2 \times 2}$ (ver [5]), en lo sucesivo sólo estudiaremos campos vectoriales holomorfos con singularidad aislada en $0 \in \mathbb{C}^{2}$ y con parte lineal no nula del tipo

$$
D Z(0)=\left[\begin{array}{cc}
\lambda_{1} & 0 \\
0 & \lambda_{2}
\end{array}\right] \in \mathbb{C}^{2 \times 2}
$$

donde $\lambda_{1}, \lambda_{2} \in \mathbb{C}^{*}$, es decir, campos de la forma

$$
Z(z)=\left(\lambda_{1} z_{1}+\sum_{|Q| \geq 2} a_{1, Q} z^{Q}, \lambda_{2} z_{2}+\sum_{|Q| \geq 2} a_{2, Q} z^{Q}\right), \quad \forall Z=\left(z_{1}, z_{2}\right) \in V
$$


donde $V$ es una vecindad abierta (polidisco) del $0 \in \mathbb{C}^{2}$ tal que $Z(z) \neq(0,0), \forall z \in V-\{0\}$. Como sabemos, este campo define el PVI

$$
\mid \begin{aligned}
& z_{1}^{\prime}=\lambda_{1} z_{1}+\sum_{|Q| \geq 2} a_{1, Q} z^{Q}, \quad z_{1}(0)=z_{1}^{0} \\
& z_{2}^{\prime}=\lambda_{2} z_{2}+\sum_{|Q| \geq 2} a_{2, Q} z^{Q}, \quad z_{2}(0)=z_{2}^{0}
\end{aligned}
$$

donde $z_{0}=\left(z_{1}^{0}, z_{2}^{0}\right) \in V$.

Denotemos por $\varphi_{Z}: D_{\delta}(0) \times \Delta\left(0, r^{\prime}\right) \rightarrow \Delta(0, r)$ el flujo local de $Z$ alrededor del 0 . Dado $z=\left(z_{1}, z_{2}\right) \in \Delta\left(0, r^{\prime}\right)$ definimos la órbita de z bajo $Z$ como

$$
\mathcal{O}_{Z}(z)=\left\{\varphi_{Z}(T, z) ; T \in \mathbb{C}\right\}
$$

y denotemos por

$$
\mathcal{F}_{Z}=\left\{\mathcal{O}_{Z}(z) ; z \in \Delta\left(0, r^{\prime}\right)\right\}
$$

a la foliación local alrededor del origen generada por $Z$. Observe que $\varphi_{Z}(T, z)$ se reduce a un punto si y sólo si $z=0$. De esta manera, si $z \neq 0$ entonces $\mathcal{O}_{Z}(z)$ es una curva.

\section{Conjugación formal y resonancias}

Sea $U \subseteq \mathbb{C}^{2}$ abierto, $0 \in U$ y $Z \in \mathcal{X}(U)$ con singularidad aislada en $0 \in \mathbb{C}^{2}$ y con parte lineal no nula del tipo $D Z(0)=\left[\begin{array}{cc}\lambda_{1} & 0 \\ 0 & \lambda_{2}\end{array}\right]$, con $\lambda_{1}, \lambda_{2} \in \mathbb{C}^{*}$. La EDO asociada sería

$$
\mid \begin{aligned}
& z_{1}^{\prime}=\lambda_{1} z_{1}+A_{1}(z) \\
& z_{2}^{\prime}=\lambda_{2} z_{2}+A_{2}(z)
\end{aligned}
$$

donde $A_{j}(z)=\sum_{|Q| \geq 2} a_{j, Q} z^{Q}, j=1,2$.

Consideremos un cambio de coordenadas (formal) del tipo perturbación de la identidad

$$
z=\left(z_{1}, z_{2}\right)=\left(w_{1}+\xi_{1}(w), w_{2}+\xi_{2}(w)\right)=\xi(w)
$$

donde $\xi_{j}(w)=\sum_{|Q| \geq 2} \xi_{j, Q} w^{Q}, j=1,2$; que transforme (3) en

$$
\mid \begin{aligned}
& w_{1}^{\prime}=\lambda_{1} w_{1}+B_{1}(w) \\
& w_{2}^{\prime}=\lambda_{2} w_{2}+B_{2}(w)
\end{aligned}
$$

donde $B_{j}(w)=\sum_{|Q| \geq 2} b_{j, Q} w^{Q}, j=1,2$.

Como $z_{j}=w_{j}+\xi_{j}(w)$, tenemos:

$$
\lambda_{j} z_{j}+A_{j}(z)=z_{j}^{\prime}=w_{j}^{\prime}+\sum_{k=1}^{2} \frac{\partial \xi_{j}}{\partial w_{k}}(w) w_{k}^{\prime}=\lambda_{j} w_{j}+B_{j}(w)+\sum_{k=1}^{2} \frac{\partial \xi_{j}}{\partial w_{k}}(w)\left(\lambda_{k} w_{k}+B_{k}(w)\right)
$$

luego

$$
\lambda_{j} w_{j}+\lambda_{j} \xi_{j}(w)+A_{j}(\xi(w))=\lambda_{j} w_{j}+B_{j}(w)+\sum_{k=1}^{2} \lambda_{k} w_{k} \frac{\partial \xi_{j}}{\partial w_{k}}(w)+\sum_{k=1}^{2} \frac{\partial \xi_{j}}{\partial w_{k}}(w) B_{k}(w)
$$


cancelando y reordenando

$$
\lambda_{j} \xi_{j}(w)-\sum_{k=1}^{2} \lambda_{k} w_{k} \frac{\partial \xi_{j}}{\partial w_{k}}(w)-B_{j}(w)=\sum_{k=1}^{2} \frac{\partial \xi_{j}}{\partial w_{k}}(w) B_{k}(w)-A_{j}(\xi(w))
$$

Como $w_{k} \frac{\partial \xi_{j}}{\partial w_{k}}(w)=\sum_{|Q| \geq 2} q_{k} \xi_{j, Q} w^{Q}$, tenemos

$$
\begin{aligned}
\lambda_{j} \xi_{j}(w)-\sum_{k=1}^{2} \lambda_{k} w_{k} \frac{\partial \xi_{j}}{\partial w_{k}}(w)-B_{j}(w)= & \sum_{|Q| \geq 2} \lambda_{j} \xi_{j, Q} w^{Q}-\sum_{k=1}^{2} \lambda_{k}\left(\sum_{|Q| \geq 2} q_{k} \xi_{j, Q} w^{Q}\right)- \\
& -\sum_{|Q| \geq 2} b_{j, Q} w^{Q} \\
= & \sum_{|Q| \geq 2}\left[\left(\lambda_{j}-\sum_{k=1}^{2} \lambda_{k} q_{k}\right) \xi_{j, Q}-b_{j, Q}\right] w^{Q}
\end{aligned}
$$

Haciendo $\delta_{j, Q}=\lambda_{j}-\sum_{k=1}^{2} \lambda_{k} q_{k}$ y reemplazando en (5) tenemos para $j=1,2$

$$
\begin{aligned}
\sum_{|Q| \geq 2}\left(\delta_{j, Q} \xi_{j, Q}-b_{j, Q}\right) w^{Q} & =\frac{\partial \xi_{j}}{\partial w_{1}}(w) B_{1}(w)+\frac{\partial \xi_{j}}{\partial w_{2}}(w) B_{2}(w)-A_{j}(\xi(w)) \\
& =\sum_{|Q| \geq 2} c_{j, Q} w^{Q}
\end{aligned}
$$

en donde $c_{j, Q}=a_{j, Q}$ si $-\mathrm{Q}-=2$ y $c_{j, Q}$ es función de $a_{1, Q^{\prime}}, a_{2, Q^{\prime}}, b_{1, Q^{\prime}}, b_{2, Q^{\prime}}, \xi_{1, Q^{\prime}}, \xi_{2, Q^{\prime}}$ (con $\left.\left|Q^{\prime}\right|<|Q|\right)$ si $|Q|>2$.

De (6), para $|Q|=2$ tenemos $\delta_{j, Q} \xi_{j, Q}-b_{j, Q}=a_{j, Q}(j=1,2)$.

Si $\delta_{j, Q}=0$ entonces hacemos $\xi_{j, Q}=0$ y por lo tanto $b_{j, Q}=-a_{j, Q}$.

Si $\delta_{j, Q} \neq 0$ entonces hacemos $b_{j, Q}=0$ y por lo tanto $\xi_{j, Q}=\frac{a_{j, Q}}{\delta_{j, Q}}$.

Para $|Q|=3$ tenemos $\delta_{j, Q} \xi_{j, Q}-b_{j, Q}=c_{j, Q}(j=1,2)$, en donde $c_{j, Q}$ depende de $a_{1, Q^{\prime}}, a_{2, Q^{\prime}}$, $b_{1, Q^{\prime}}, b_{2, Q^{\prime}}, \xi_{1, Q^{\prime}}, \xi_{2, Q^{\prime}}$ con $\left|Q^{\prime}\right|=2$. Por el paso anterior, todas estas constantes son conocidas, luego $c_{j, Q}$ es conocido y nuestras incógnitas son $\xi_{j, Q}$ y $b_{j, Q}$ que la despejamos como en el paso anterior:

Si $\delta_{j, Q}=0$ entonces hacemos $\xi_{j, Q}=0$ y por lo tanto $b_{j, Q}=-a_{j, Q}$.

Si $\delta_{j, Q} \neq 0$ entonces hacemos $b_{j, Q}=0$ y por lo tanto $\xi_{j, Q}=\frac{a_{j, Q}}{\delta_{j, Q}}$.

El proceso continúa por inducción. Resumimos nuestros resultados en el siguiente:

Teorema 3.1. Dada la EDO

$$
\mid \begin{aligned}
& z_{1}^{\prime}=\lambda_{1} z_{1}+\sum_{|Q| \geq 2} a_{1, Q} z^{Q} \\
& z_{2}^{\prime}=\lambda_{2} z_{2}+\sum_{|Q| \geq 2} a_{2, Q} z^{Q}
\end{aligned}
$$


existe un cambio formal de coordenadas del tipo perturbación de la identidad:

$$
\mid \begin{aligned}
z_{1} & =w_{1}+\sum_{|Q| \geq 2} \xi_{1, Q} w^{Q} \\
z_{2} & =w_{2}+\sum_{|Q| \geq 2} \xi_{2, Q} w^{Q}
\end{aligned}
$$

que transforma (7) en

$$
\mid \begin{aligned}
& w_{1}^{\prime}=\lambda_{1} w_{1}+\sum_{|Q| \geq 2} b_{1, Q} w^{Q} \\
& w_{2}^{\prime}=\lambda_{2} w_{2}+\sum_{|Q| \geq 2} b_{2, Q} w^{Q}
\end{aligned}
$$

en donde los coeficientes $\xi_{j, Q}$ y $b_{j, Q}$ satisfacen la relación

$$
\mid \begin{aligned}
& b_{j, Q}=0, \quad \text { si } \delta_{j, Q}=\lambda_{j}-q_{1} \lambda_{1}-q_{2} \lambda_{2} \neq 0 . \\
& \xi_{j, Q}=0, \quad \text { si } \delta_{j, Q}=0
\end{aligned}
$$

\section{Observaciones:}

1. Dada la EDO (7), el procedimiento anterior nos permite construir el cambio de coordenadas (8) y la EDO transformada (9).

2. El teorema anterior no nos da ninguna información sobre la convergencia de las series formales $\sum_{|Q| \geq 2} \xi_{j, Q} w^{Q}, \sum_{|Q| \geq 2} b_{j, Q} w^{Q}$.

3. Note la importancia de las expresiones $\delta_{j, Q}=\lambda_{j}-q_{1} \lambda_{1}-q_{2} \lambda_{2}$. En el caso de que $\delta_{j, Q}=0$, $\forall j=1,2, \forall|Q| \geq 2$, el cambio de coordenadas (8) sería la identidad y la EDO (7) sería la EDO (9) (no ganaríamos nada). El otro extremo ocurre cuando $\delta_{j, Q} \neq 0, \forall j=1,2$, $\forall|Q| \geq 2$, en este caso la EDO transformada (9) sería

$$
\mid \begin{aligned}
& w_{1}^{\prime}=\lambda_{1} w_{1} \\
& w_{2}^{\prime}=\lambda_{2} w_{2}
\end{aligned}
$$

la cual es una EDO lineal En general uno espera obtener un caso intermedio en el sentido que la EDO transformada (9) sería más simple que la EDO original (7).

La expresión (10) motiva el siguiente concepto.

Definición 3.1. Decimos que $\left(\lambda_{1}, \lambda_{2}\right) \in \mathbb{C}^{*} \times \mathbb{C}^{*}$ es un par resonante si y sólo si existen $q_{1}, q_{2} \in \mathbb{N}$, con $q_{1}+q_{2} \geq 2$ tales que

$$
\lambda_{1}=q_{1} \lambda_{1}+q_{2} \lambda_{2} \quad \delta \quad \lambda_{2}=q_{1} \lambda_{1}+q_{2} \lambda_{2}
$$

Las dos proposiciones siguientes, cuya demostración puede ser encontrada en ([2]), nos proporcionan criterios sencillos para determinar si un par es o no resonante. 
Proposición 3.1. Sea $\left(\lambda_{1}, \lambda_{2}\right) \in \mathbb{C}^{*} \times \mathbb{C}^{*}$, tales que $\frac{\lambda_{1}}{\lambda_{2}} \notin \mathbb{R}^{-}$Son equivalentes:

1. $\left(\lambda_{1}, \lambda_{2}\right)$ es un par resonante.

2. Existe un único entero $m \geq 2$ tal que $\lambda_{1}=m \lambda_{2}$ ó $\lambda_{2}=m \lambda_{1}$.

Proposición 3.2. Sea $\left(\lambda_{1}, \lambda_{2}\right) \in \mathbb{C}^{*} \times \mathbb{C}^{*}$, tales que $\frac{\lambda_{1}}{\lambda_{2}} \in \mathbb{R}^{-}$. Son equivalentes:

1. $\left(\lambda_{1}, \lambda_{2}\right)$ es un par resonante.

2. Existen $m_{1}, m_{2} \in \mathbb{Z}^{+}$tales que $m_{1} \lambda_{1}+m_{2} \lambda_{2}=0$.

Si $\left(\lambda_{1}, \lambda_{2}\right) \in \mathbb{C}^{*} \times \mathbb{C}^{*}$, tales que $\frac{\lambda_{1}}{\lambda_{2}} \notin \mathbb{R}^{-}$entonces decimos que $\left(\lambda_{1}, \lambda_{2}\right)$ está en el dominio de Poincaré, lo que denotaremos por $\left(\lambda_{1}, \lambda_{2}\right) \in D_{P}$.

Usando la noción de resonancia, podemos reformular el teorema 3.1

Teorema 3.2. Si $\left(\lambda_{1}, \lambda_{2}\right) \in \mathbb{C}^{*} \times \mathbb{C}^{*}$ es un par no resonante entonces la EDO

$$
\mid \begin{aligned}
z_{1}^{\prime} & =\lambda_{1} z_{1}+\sum_{|Q| \geq 2} a_{1, Q} z^{Q} \\
z_{2}^{\prime} & =\lambda_{2} z_{2}+\sum_{|Q| \geq 2} a_{2, Q} z^{Q}
\end{aligned}
$$

puede ser linealizada por un cambio formal de coordenadas del tipo perturbación de la identidad.

Cuando $\left(\lambda_{1}, \lambda_{2}\right) \in \mathbb{C}^{*} \times \mathbb{C}^{*}$ es un par no resonante, entonces el Teorema de Poincaré (ver [2]) afirma que el cambio de coordenadas formal de coordenadas del Teorema 3.1, es convergente en una vecindad del origen, es esto esencialmente lo que dice el Teorema de linealización.de Poincaré. Más aún, se puede tener el siguiente resultado, cuya demostración el lector la puede encontrar en ([1]):

Teorema 3.3. (Teorema de Linealización de Poincaré) Sea $U \subseteq \mathbb{C}^{2}$ abierto, $0 \in U$ y $Z \in \mathcal{X}(U)$ tal que 0 es una singularidad aislada de $Z$. Si la parte lineal de $Z$ en 0 es no nula y sus autovalores $\left(\lambda_{1}, \lambda_{2}\right)$ es un par no resonante en el dominio de Poincaré, entonces $Z$ es localmente equivalente a su parte lineal.

\section{El Resultado Principal}

Sea $U \subseteq \mathbb{C}^{2}$ abierto, $0 \in U$ y $Z \in \mathcal{X}(U)$ tal que 0 es una singularidad aislada de $Z$. Cuando la parte lineal de $Z$ en 0 es no nula y sus autovalores $\left(\lambda_{1}, \lambda_{2}\right) \in D_{P}$ es un par resonante, entonces ya no es posible transformar la EDO asociada a $Z$ en una EDO lineal, sin embargo, la situación no es tan complicada.

Teorema 4.1. (Dulac) Sea $U \subseteq \mathbb{C}^{2}$ abierto, $0 \in U$ y $Z \in \mathcal{X}(U)$ tal que 0 es una singularidad aislada de $Z$. Si la parte lineal de $Z$ en 0 es no nula y sus autovalores $\left(\lambda_{1}, \lambda_{2}\right)$ es un par resonante en el dominio de Poincaré, entonces $Z$ es localmente equivalente al campo $W \in \mathcal{X}\left(\mathbb{C}^{2}\right)$ definido por

$$
W\left(w_{1}, w_{2}\right)=\left(\lambda_{1} w_{1}+a w_{2}^{m}, \lambda_{2} w_{2}\right) \quad\left(\operatorname{resp} . W\left(w_{1}, w_{2}\right)=\left(\lambda_{1} w_{1}, \lambda_{2} w_{2}+b w_{1}^{m}\right)\right)
$$

en donde el entero $m \geq 2$ es tal que $\lambda_{1}=m \lambda_{2}$ (resp. $\left.\lambda_{2}=m \lambda_{1}\right)$. 
Prueba. De acuerdo a (6), tenemos que existe un cambio formal de coordenadas

$$
\sum_{|Q| \geq 2}\left(\delta_{j, Q} \xi_{j, Q}-b_{j, Q}\right) w^{Q}=\frac{\partial \xi_{j}}{\partial w_{1}}(w) B_{1}(w)+\frac{\partial \xi_{j}}{\partial w_{2}}(w) B_{2}(w)-A_{j}(\xi(w))
$$

Como por hipótesis $\left(\lambda_{1}, \lambda_{2}\right) \in D_{P}$ es un par resonante, luego, por la Proposición 3.1, existe un único entero $m \geq 2$ tal que $\lambda_{1}=m \lambda_{2}$ ó $\lambda_{2}=m \lambda_{1}$. Trabajando con el primer caso y denotando $Q_{0}=(0, m)$, 'tenemos que $\delta_{1, Q} \neq 0, \forall|Q| \geq 2, Q \neq Q_{0}$ y $\delta_{2, Q} \neq 0, \forall|Q| \geq 2$. Se tiene entonces que

$$
B_{1}(w)=\sum_{|Q| \geq 2} b_{1, Q} w^{Q}=b_{1, Q_{0}} w^{Q_{0}} \quad \text { y } \quad B_{2}(w)=\sum_{|Q| \geq 2} b_{2, Q} w^{Q}=0
$$

Además como $\delta_{1, Q_{0}}=\lambda_{1}-0 \cdot \lambda_{1}-m \lambda_{2}=0$ entonces $\xi_{1, Q_{0}}=0$. Reemplazando en la igualdad inicial tenemos para $j=1$ :

$$
\sum_{|Q| \geq 2} \delta_{1, Q} \xi_{1, Q} w^{Q}-b_{1, Q_{0}} w^{Q_{0}}=\frac{\partial \xi_{1}}{\partial w_{1}}(w) b_{1, Q_{0}} w^{Q_{0}}-A_{1}(\xi(w))
$$

mientras que para $j=2$ :

$$
\sum_{|Q| \geq 2} \delta_{2, Q} \xi_{2, Q} w^{Q}=\frac{\partial \xi_{2}}{\partial w_{1}}(w) b_{1, Q_{0}} w^{Q_{0}}-A_{2}(\xi(w))
$$

Como $\xi_{j}(w)=\sum_{|Q| \geq 2} \xi_{j, Q} w^{Q}$ entonces $\frac{\partial \xi_{j}}{\partial w_{1}}(w)=\sum_{|Q| \geq 2} \xi_{j, Q} q_{1} w_{1}^{q_{1}-1} w_{2}^{q_{2}}$, luego

$$
w_{1} \frac{\partial \xi_{j}}{\partial w_{1}}(w)=\sum_{|Q| \geq 2} \xi_{j, Q} q_{1} w^{Q} \Rightarrow \frac{\partial \xi_{j}}{\partial w_{1}}(w)=\sum_{|Q| \geq 2} \xi_{j, Q} \frac{q_{1}}{w_{1}} w^{Q}
$$

y por tanto

$$
\frac{\partial \xi_{j}}{\partial w_{1}}(w) b_{1, Q_{0}} w^{Q_{0}}=\sum_{|Q| \geq 2} q_{1} b_{1, Q_{0}} \frac{w_{2}^{m}}{w_{1}} \xi_{j, Q} w^{Q}
$$

Reemplazando y ordenando obtenemos:

$$
\begin{gathered}
\sum_{|Q| \geq 2}\left(\delta_{1, Q}-q_{1} b_{1, Q_{0}} \frac{w_{2}^{m}}{w_{1}}\right) \xi_{1, Q} w^{Q}=b_{1, Q_{0}} w^{Q_{0}}-A_{1}(\xi(w)) \\
\sum_{|Q| \geq 2}\left(\delta_{2, Q}-q_{1} b_{1, Q_{0}} \frac{w_{2}^{m}}{w_{1}}\right) \xi_{2, Q} w^{Q}=-A_{2}(\xi(w))
\end{gathered}
$$

Denotando $P_{j}\left(w_{1}, w_{2}\right)=\sum_{|Q| \geq 2}\left(\delta_{j, Q}-q_{1} b_{1, Q_{0}} \frac{w_{2}^{m}}{w_{1}}\right) \xi_{j, Q} w^{Q}$, se tiene

$$
P_{1}\left(w_{1}, w_{2}\right)=b_{1, Q_{0}} w^{Q_{0}}-A_{1}(\xi(w)) \quad \text { y } \quad P_{2}\left(w_{1}, w_{2}\right)=-A_{2}(\xi(w))
$$

AFIRMACIÓN: $\hat{A}_{j}(\xi(w)) \leq \hat{A}_{j}\left(w_{1}+\hat{\xi}_{1}, w_{2}+\hat{\xi}_{2}(w)\right)$. En efecto, como

$$
\begin{aligned}
\hat{A}_{j}(\xi(w)) & =\sum_{|Q| \geq 2}\left|a_{j, Q}\right|\left(w_{1}+\xi_{1}(w)\right)^{q_{1}}\left(w_{2}+\xi_{2}(w)\right)^{q_{2}} \\
& \leq \sum_{|Q| \geq 2}\left|a_{j, Q}\right|\left(w_{1}+\hat{\xi}_{1}(w)\right)^{q_{1}}\left(w_{2}+\hat{\xi}_{2}(w)\right)^{q_{2}}=\hat{A}_{j}\left(w_{1}+\hat{\xi}_{1}, w_{2}+\hat{\xi}_{2}(w)\right)
\end{aligned}
$$


Entonces:

$$
\begin{gathered}
\hat{P}_{1}\left(w_{1}, w_{2}\right) \leq\left|b_{1, Q_{0}}\right| w^{Q_{0}}+A_{1}(\hat{\xi}(w)) \leq\left|b_{1, Q_{0}}\right| w^{Q_{0}}+\hat{A}_{1}\left(w_{1}+\hat{\xi}_{1}, w_{2}+\hat{\xi}_{2}(w)\right) \\
\hat{P}_{2}\left(w_{1}, w_{2}\right)=\hat{A}_{2}(\xi(w)) \leq \hat{A}_{2}\left(w_{1}+\hat{\xi}_{1}(w), w_{2}+\hat{\xi}_{2}(w)\right)
\end{gathered}
$$

Tomando $w_{1}=w_{2}=w$ tenemos

$$
\tilde{P}_{1}(w)=\hat{P}_{1}(w, w) \leq\left|b_{1, Q_{0}}\right| w^{m}+\hat{A}_{1}\left(w+\tilde{\xi}_{1}(w), w+\tilde{\xi}_{2}(w)\right)
$$

y

$$
\tilde{P}_{2}(w)=\hat{P}_{2}(w, w) \leq \hat{A}_{2}\left(w+\tilde{\xi}_{1}(w), w+\tilde{\xi}_{2}(w)\right)
$$

Sumando ambas expresiones tenemos

$$
\begin{aligned}
\tilde{P}_{1}(w)+\tilde{P}_{2}(w) & \leq\left|b_{1, Q_{0}}\right| w^{m}+\hat{A}_{1}\left(w+\tilde{\xi}_{1}, w+\tilde{\xi}_{2}\right)+\hat{A}_{2}\left(w+\tilde{\xi}_{1}, w+\tilde{\xi}_{2}\right) \\
& \leq\left|b_{1, Q_{0}}\right| w^{m}+\hat{A}_{1}\left(w+\tilde{\xi}_{1}+\tilde{\xi}_{2}, w+\tilde{\xi}_{1}+\tilde{\xi}_{2}\right)+\hat{A}_{2}\left(w+\tilde{\xi}_{1}+\tilde{\xi}_{2}, w+\tilde{\xi}_{1}+\tilde{\xi}_{2}\right) \\
& =\left|b_{1, Q_{0}}\right| w^{m}+\tilde{A}_{1}\left(w+\tilde{\xi}_{1}+\tilde{\xi}_{2}\right)+\tilde{A}_{2}\left(w+\tilde{\xi}_{1}+\tilde{\xi}_{2}\right)
\end{aligned}
$$

En conclusión tenemos

$$
\tilde{P}_{1}(w)+\tilde{P}_{2}(w) \leq\left|b_{1, Q_{0}}\right| w^{m}+\tilde{A}_{1}\left(w+\tilde{\xi}_{1}+\tilde{\xi}_{2}\right)+\tilde{A}_{2}\left(w+\tilde{\xi}_{1}+\tilde{\xi}_{2}\right)
$$

Luego:

$$
\sum_{j=1}^{2} \tilde{P}_{j}(w) \leq\left|b_{1, Q_{0}}\right| w^{m}+\sum_{k=1}^{2} \tilde{A}_{k}\left(w+\sum_{j=1}^{2} \tilde{\xi}_{j}(w)\right)
$$

Por otro lado, recordemos que

$$
\tilde{P}_{j}(w)=\sum_{|Q| \geq 2}\left|\delta_{j, Q}-q_{1} b_{1, Q_{0}} w^{m-1}\right| \cdot\left|\xi_{j, Q}\right| w^{|Q|}
$$

Antes de continuar, necesitamos el siguiente resultado.

Lema 4.1. Para $\epsilon>0$ suficientemente pequeño tenemos que

$$
\text { inf }\left\{\left|\left(\delta_{j, Q}-q_{1} b_{1, Q_{0}}\right) w^{m-1}\right| ; \quad \forall(j, Q) \neq\left(1, Q_{0}\right) \text { y }|w|<\epsilon\right\}>0
$$

Prueba. Tomando $\epsilon=\left|\frac{2 \lambda_{2}}{b_{1, Q_{0}}}\right|^{\frac{1}{m-1}}>0$, tenemos que si $|w|<\epsilon$ entonces $\left|\frac{b_{1, Q_{0}} w^{m-1}}{\lambda_{2}}\right|<\frac{1}{2}$. Sea $K=\operatorname{Re}\left(\frac{b_{1, Q_{0} w^{m-1}}}{\lambda_{2}}\right) \Rightarrow|K|<\frac{1}{2}$. Consideremos dos casos:

Caso 1: $j=2$. Entonces $(j, Q) \neq\left(1, Q_{0}\right)$, luego

$$
\begin{aligned}
\left|S_{j, Q}-q_{1} b_{1, Q_{0}} w^{m-1}\right| & =\left|\lambda_{2}-q_{1} \lambda_{1}-q_{2} \lambda_{2}-q_{1} b_{1, Q_{0}} w^{m-1}\right|=\left|\lambda_{2}\right| \cdot\left|1-q_{1} m-q_{2}-q_{1} \frac{b_{1, Q_{0}}}{\lambda_{2}} w^{m-1}\right| \\
& \geq\left|\lambda_{2}\right| \cdot\left|\operatorname{Re}\left(1-q_{1} m-q_{2}-q_{1} \frac{b_{1, Q_{0}}}{\lambda_{2}} w^{m-1}\right)\right|=\left|\lambda_{2}\right| \cdot\left|1-q_{1} m-q_{2}-q_{1} K\right| \\
& \geq\left|\lambda_{2}\right|\left(q_{1} m+q_{1} K+q_{2}-1\right) \geq\left|\lambda_{2}\right|\left(q_{1}(m+K)+q_{2}-1\right) \\
& \geq\left|\lambda_{2}\right|\left(q_{1}\left(2-\frac{1}{2}\right)+q_{2}-1\right)=\left|\lambda_{2}\right|\left(\frac{3}{2} q_{1}+q_{2}-1\right) \\
& \geq\left|\lambda_{2}\right|\left(q_{1}+q_{2}-1\right) \geq\left|\lambda_{2}\right|
\end{aligned}
$$


Caso 2: $j=1$. Entonces $Q \neq Q_{0}=(0, m)$. Analicemos el valor de $\left|m-q_{1} m-q_{2}-q_{1} K\right|$

Si $q_{1}=0$ entonces $Q_{0} \neq Q$ lo cual implica que $q_{2} \neq m$ y por tanto

$$
\left|m-q_{1} m-q_{2}-q_{1} K\right|=\left|m-q_{2}\right| \geq 1
$$

Si $q_{1}=1$, tenemos

$$
\begin{aligned}
\left|m-q_{1} m-q_{2}-q_{1} K\right| & =\left|q_{2}+K\right| \geq q_{2}+K \geq q_{2}-\frac{1}{2} \geq q_{1}+q_{2}-\frac{1}{2}-1 \\
& \geq 2-\frac{3}{2}=\frac{1}{2}
\end{aligned}
$$

Si $q_{1} \geq 2$, tenemos

$$
\begin{aligned}
\left|m-q_{1} m-q_{2}-q_{1} K\right| & \geq q_{1} m+q_{2}+q_{1} K-m=m\left(q_{1}-1\right)+q_{2}+q_{1} K \\
& \geq 2\left(q_{1}-1\right)+q_{2}-\frac{q_{1}}{2}=\frac{3 q_{1}}{2}-2+q_{2}=q_{1}+q_{2}-2+\frac{q_{1}}{2} \\
& \geq \frac{q_{1}}{2} \geq 1
\end{aligned}
$$

Entonces

$$
\begin{aligned}
\left|\delta_{j, Q}-q_{1} b_{1, Q_{0}} w^{m-1}\right| & =\left|\lambda_{1}-q_{1} \lambda_{1}-q_{2} \lambda_{2}-q_{1} b_{1, Q_{0}} w^{m-1}\right|=\left|\lambda_{2}\right| \cdot\left|m-q_{1} m-q_{2}-q_{1} \frac{b_{1, Q_{0}}}{\lambda_{2}} w^{m-1}\right| \\
& \geq\left|\lambda_{2}\right| \cdot\left|R e\left(m-q_{1} m-q_{2}-q_{1} \frac{b_{1, Q_{0}}}{\lambda_{2}} w^{m-1}\right)\right|=\left|\lambda_{2}\right| \cdot\left|m-q_{1} m-q_{2}-q_{1} K\right| \\
& \geq \frac{\left|\lambda_{2}\right|}{2}
\end{aligned}
$$

En conclusión, si $\epsilon=\left|\frac{2 \lambda_{2}}{b_{1, Q_{0}}}\right|^{\frac{1}{m-1}}$, entonces

$$
\inf \left\{\left|\delta_{j, Q}-q_{1} b_{1, Q_{0}} w^{m-1}\right| ; \quad(j, Q) \neq\left(1, Q_{0}\right) \quad \text { y } \quad|w|<\epsilon\right\} \geq \frac{\left|\lambda_{2}\right|}{2}>0
$$

lo que concluye la demostración del lema.

Volviendo a la demostración del Teorema de Dulac, en (12) apliquemos el lema:

$$
\tilde{P}_{j}(w)=\sum_{|Q| \geq 2}\left|\delta_{j, Q}-q_{1} b_{1, Q_{0}} w^{m-1}\right| \cdot\left|\xi_{j, Q}\right| w^{|Q|} \geq \sum_{|Q| \geq 2} \frac{\left|\lambda_{2}\right|}{2}\left|\xi_{j, Q}\right| w^{|Q|}=\frac{\left|\lambda_{2}\right|}{2} \tilde{\xi}_{j}(w)
$$

Esto es válido puesto cuando $(j, Q)=\left(1, Q_{0}\right)$ tenemos que $\tilde{\xi}_{1, Q_{0}}=0$, luego

$$
\sum_{j=1}^{2} \tilde{P}_{j}(w) \geq \frac{\left|\lambda_{2}\right|}{2} \sum_{j=1}^{2} \tilde{\xi}_{j}(w)
$$

Usando (12) y (13), tenemos

$$
\frac{\left|\lambda_{2}\right|}{2} \sum_{j=1}^{2} \tilde{\xi}_{j}(w) \leq\left|b_{1, Q_{0}}\right| w^{m}+\sum_{j=1}^{2} \tilde{A}_{j}\left(w+\sum_{j=1}^{2} \tilde{\xi}_{j}(w)\right)
$$

Denotando $b=\frac{2\left|b_{1, Q_{0}}\right|}{\left|\lambda_{2}\right|}, S(w)=\sum_{j=1}^{2} \tilde{\xi}_{j}(w)$ y $F(w)=\frac{2}{\left|\lambda_{2}\right|} \sum_{j=1}^{2} \tilde{A}_{j}(w)$, tenemos 


$$
S(w) \leq b w^{n}+F(w+S(w)) ; \forall|w|<\epsilon=\left|\frac{2 \lambda_{2}}{b_{1, Q_{0}}}\right|^{\frac{1}{m-1}}
$$

Como $S(w) \leq F(w+S(w))+b w^{m}$, en una vecindad del $0 \in \mathbb{C}^{2}$, definimos $f$ a valores complejos como:

$$
f(w, v)=v-F(w+v)-b w^{m}
$$

Observe que

$$
f(0,0)=0 \quad \text { y } \quad \frac{\partial f}{\partial v}(w, v)=1-F^{\prime}(w+v)
$$

luego $\frac{\partial f}{\partial v}(0,0)=1 \neq 0$. Por el Teorema de la función implícita para funciones de varias variables complejas (ver [3]) existe una función analítica $\varphi: D_{R}(0) \rightarrow D_{R^{\prime}}(0)$ tal que

$$
R<\epsilon, \quad \varphi(0)=0 \quad \text { y } \quad f(w, \varphi(w))=0, \quad \forall w \in D_{R}(0) .
$$

Luego

$$
\varphi(w)=F(w+\varphi(w))+b w^{m}, \quad \forall w \in \dot{D_{R}}(0)
$$

Además

$$
\begin{aligned}
\varphi^{\prime}(w) & =F^{\prime}(w+\varphi(w))\left(1+\varphi^{\prime}(w)\right)+b m w^{m-1}, \quad \forall w \in D_{R}(0) \\
\varphi^{\prime}(0) & =F^{\prime}(0+\varphi(0))\left(1+\varphi^{\prime}(0)\right)=0
\end{aligned}
$$

Por lo tanto:

$$
\varphi(w)=\sum_{n \geq 2} c_{n} w^{n} ; \quad \forall w \in D_{R}(0)
$$

Luego tenemos

$$
\sum_{n \geq 2} c_{n} w^{n}=\sum_{n \geq 1} f_{n}\left(w+c_{2} w^{2}+c_{3} w^{3}+\ldots\right)^{n}+b w^{m}
$$

Igualando términos de orden " $n$ "

$$
\begin{array}{llll}
c_{2} & =f_{2} \quad \text { ó } & c_{2}=f_{2}+b \\
c_{3} & =2 c_{2} f_{2} \quad \text { ó } & c_{3}=2 c_{2} f_{2}+b \\
c_{4} & = & c_{2}^{2} f_{2}+2 f_{2} c_{3}+2 f_{3} c_{2}+f_{4}
\end{array}
$$

se sigue que $c_{n} \geq f_{n} \forall n \geq 2, \varphi(w)$ es una serie de términos no negativos. Por otro lado, como

$$
S(w) \leq F(w+S(w))+b w^{m}
$$

se tiene entonces que

$$
\begin{aligned}
& S_{2} \leq f_{2} \quad \text { ó } \quad S_{2} \leq f_{2}+b=c_{2}+b \\
& S_{3} \leq 2 S_{2} f_{2} \leq 2 c_{2} f_{2}=c_{3} \quad \text { ó } \quad S_{3} \leq c_{3}+b \\
& S_{4} \leq S_{2}^{2} f_{2}+2 f_{2} S_{3}+2 f_{3} S_{2}+f_{4} \leq c_{2}^{2} f_{2}+2 f_{2} c_{3}+2 f_{3} c_{2}+f_{4}=c_{4}
\end{aligned}
$$

Prosiguiendo tenemos $S_{n} \leq c_{n}, \quad \forall n \geq 2$. 
Como $\sum_{n \geq 2} c_{n} w^{n}$ es convergente entonces $S(w)=\sum_{n \geq 2} S_{n} w^{n}$ es convergente en $D_{R}(0)$, luego $\tilde{\xi}_{1}(w)+\tilde{\xi}_{2}(w)$ es convergente en $D_{R}(0)$ y por tanto $\tilde{\xi}_{1}(w), \tilde{\xi}_{2}(w)$ son convergentes en $D_{R}(0)$. Se sigue que $\xi_{1}\left(w_{1}, w_{2}\right)$ y $\xi_{2}\left(w_{1}, w_{2}\right)$ son convergentes en el polidisco $\triangle((0,0),(R, R))$. Esto prueba que el cambio de coordenadas es convergente.

Para demostrar la conjugación, sea $\xi(w)=\left(w_{1}+\xi_{1}(w), w_{2}+\xi_{2}(w)\right)$ el cambio de coordenadas tipo perturbación de la identidad que transforma la EDO asociada a $Z$ a

$$
\left(\lambda_{1} w_{1}+b_{1,(0, m)} w_{2}^{m}, \lambda_{2} w_{2}\right)
$$

Sabemos que $\xi$ es holomorfa en una vecindad del 0 y $\xi^{\prime}(0,0)=\left[\begin{array}{ll}1 & 0 \\ 0 & 1\end{array}\right] \in G L\left(\mathbb{C}^{2}\right)$. Por el Teorema de la función inversa para funciones de varias variables complejas (ver [3]), existen polidiscos abiertos $\triangle_{1}, \triangle_{2} \subseteq \mathbb{C}^{2}$ centrados en $0 \in \mathbb{C}^{2}$ tales que

$$
\left.\xi\right|_{\triangle_{1}}: \triangle_{1} \rightarrow \triangle_{2} \text { es un biholomorfismo }
$$

Si denotamos $h=\left(\left.\xi\right|_{\triangle_{1}}\right)^{-1} \operatorname{como} w=h(z)$ se tiene que $w^{\prime}=h^{\prime}(z) z^{\prime}$ implica $\left(\lambda_{1} w_{1}+b_{1, Q_{0}} w_{2}^{m}, \lambda_{2} w_{2}\right)=h^{\prime}(z) Z(z)$ y por tanto $W(w)=h^{\prime}(z) Z(z)$. Por la Proposición 1.1, concluimos que $h$ es una conjugación analítica entre $Z$ y $W$. Esto finaliza la prueba del Teorema de Dulac.

\section{Estudio geométrico local}

De acuerdo al teorema de Dulac, para entender el comportamiento geométrico de las soluciones de una EDO definida por un campo cuya parte lineal tiene autovalores resonantes en el dominio de Poincaré, es suficiente entender las soluciones del campo polinomial $Z\left(z_{1}, z_{2}\right)=\left(\lambda_{1} z_{1}+a z_{2}^{m}, \lambda_{2} z_{2}\right)$. Vamos a hacer este estudio a continuación. En primer lugar, un fácil cálculo muestra que la solución $\varphi_{z_{0}}: \mathbb{C} \rightarrow \mathbb{C}^{2}$ del PVI asociado al campo $Z$ que en el instante 0 pasa por $z_{0}=\left(z_{1}^{0}, z_{2}^{0}\right)$, viene dada por

$$
\varphi_{z_{0}}(T)=\left(\left(a\left(z_{2}^{0}\right)^{m} T+z_{1}^{0}\right) e^{\lambda_{1} T}, z_{2}^{0} e^{\lambda_{2} T}\right)
$$

De esta manera, el flujo (global) $\varphi: \mathbb{C} \times \mathbb{C}^{2} \rightarrow \mathbb{C}^{2}$ asociado a $Z$ se define como

$$
\varphi\left(T, z_{1}, z_{2}\right)=\left(\left(z_{1}+a\left(z_{2}\right)^{m} T\right) e^{\lambda_{1} T}, z_{2} e^{\lambda_{2} T}\right)
$$

Nos proponemos estudiar la foliación $\mathcal{F}_{Z}$. En primer lugar, observe que $(0,0) \in \mathbb{C}^{2}$ es la única singularidad de $Z$, luego $\mathcal{O}_{Z}((0,0))=\{(0,0)\}$. Además, es fácil ver que $\mathcal{O}_{Z}((z, 0))=\mathbb{C}^{*} \times\{0\}$. A diferencia de lo que ocurre con las lineales $\{0\} \times \mathbb{C}^{*} \notin \mathcal{F}_{Z}$.

Sea $L \in \mathcal{F}_{Z}-\{(0,0)\}$, vamos a probar que $L$ intersecta transversalmente a la familia de esferas $\left\{S_{r}^{3}\right\}_{r>0}$, para $r>0$ suficientemente pequeño. En efecto, sabemos que el espacio tangente a $S_{r}^{3}=\left\{\left(z_{1}, z_{2}\right) \in \mathbb{C}^{2} ;\left|z_{1}\right|^{2}+\left|z_{2}\right|^{2}=r^{2}\right\}$ en el punto $z_{0}=\left(z_{1}^{0}, z_{2}^{0}\right) \in S_{r}^{3}$ es

$$
T_{z_{0}}=\left\{z \in \mathbb{C}^{2} ; \operatorname{Re}\left(\left\langle z, z_{0}\right\rangle\right)=0\right\}
$$

Como $\left(\lambda_{1}, \lambda_{2}\right) \in \mathcal{D}_{P}$, existe $\alpha \in S^{1}$ tal que $\operatorname{Re}\left(\alpha \lambda_{1}\right)<0$ y $\operatorname{Re}\left(\alpha \lambda_{2}\right)<0$, además, como $\left(\lambda_{1}, \lambda_{2}\right) \in \mathcal{D}_{P}$ es un par resonante, se tiene que existe $m \geq 2$ tal que $\lambda_{1}=m \lambda_{2}$. Sea $L \in \mathcal{F}_{Z}-\{(0,0)\}$, podemos considerar

$$
L=\mathcal{O}_{Z}\left(z_{0}\right)=\left\{\varphi_{z_{0}}(T) ; T \in \mathbb{C}\right\}
$$


Sabemos que

$$
T_{z_{0}} L=\left\{c \varphi_{z_{0}}^{\prime}(0) ; c \in \mathbb{C}\right\}=\left\{c\left(\lambda_{1} z_{1}^{0}+a\left(z_{2}^{0}\right)^{m}, \lambda_{2} z_{2}^{0}\right) ; c \in \mathbb{C}\right\}
$$

Tomando $c=\alpha$ tenemos:

$$
\begin{aligned}
\operatorname{Re}\left(\left\langle\alpha \varphi_{z_{0}}^{\prime}(0), z_{0}\right\rangle\right) & =\operatorname{Re}\left(\left\langle\alpha\left[\lambda_{1} z_{1}^{0}+a\left(z_{2}^{0}\right)^{m}, \lambda_{2} z_{2}^{0}\right],\left(z_{1}^{0}, z_{2}^{0}\right)\right\rangle\right) \\
& =\operatorname{Re}\left(\alpha\left[\lambda_{1} z_{1}^{0}+a\left(z_{2}^{0}\right)^{m}\right] \bar{z}_{1}^{0}+\alpha \lambda_{2} z_{2}^{0} \bar{z}_{2}^{0}\right) \\
& =\operatorname{Re}\left(\alpha \lambda_{1}\left|z_{1}^{0}\right|^{2}+\alpha a\left(z_{2}^{0}\right)^{m} \bar{z}_{1}^{0}+\alpha \lambda_{2}\left|z_{2}^{0}\right|^{2}\right) \\
& =\left|z_{1}^{0}\right|^{2} \operatorname{Re}\left(\alpha \lambda_{1}\right)+\operatorname{Re}\left(\alpha a\left(z_{2}^{0}\right)^{m} \bar{z}_{1}^{0}\right)+\left|z_{2}^{0}\right|^{2} \operatorname{Re}\left(\alpha \lambda_{2}\right) \\
& =\left(m\left|z_{1}^{0}\right|^{2}+\left|z_{2}^{0}\right|^{2}\right) \operatorname{Re}\left(\alpha \lambda_{2}\right)+\operatorname{Re}\left(\alpha a\left(z_{2}^{0}\right)^{m} \bar{z}_{1}^{0}\right) \\
& \leq\left(m\left|z_{1}^{0}\right|^{2}+\left|z_{2}^{0}\right|^{2}\right) \operatorname{Re}\left(\alpha \lambda_{2}\right)+|a| \cdot\left|z_{1}^{0}\right| \cdot\left|z_{2}^{0}\right|^{m}
\end{aligned}
$$

Ahora bien, como $\left|z_{1}^{0}\right|^{2}+\left|z_{2}^{0}\right|^{2}=r^{2}$ entonces $\left|z_{1}^{0}\right|^{2} \leq r^{2}$ y $\left|z_{2}^{0}\right|^{2} \leq r^{2}$, luego

$$
|a| \cdot\left|z_{1}^{0}\right| \cdot\left|z_{2}^{0}\right|^{m} \leq|a| r^{m+1} .
$$

Además $m\left|z_{1}^{0}\right|^{2}+\left|z_{2}^{0}\right|^{2}=(m-1)\left|z_{1}^{0}\right|^{2}+r^{2} \geq r^{2}$, luego

$$
\left[m\left|z_{1}^{0}\right|^{2}+\left|z_{2}^{0}\right|^{2}\right] \operatorname{Re}\left(\alpha \lambda_{2}\right) \leq r^{2} \operatorname{Re}\left(\alpha \lambda_{2}\right)^{\circ}
$$

Reemplazando estas dos desigualdades en la anterior se llega a

$$
\operatorname{Re}\left(\left\langle\alpha \varphi_{z_{0}}^{\prime}(0), z_{0}\right\rangle\right) \leq r^{2} \operatorname{Re}\left(\alpha \lambda_{2}\right)+|a| r^{m+1}
$$

Tomando $r>0$ tal que $r^{m-1}<-\frac{\operatorname{Re}\left(\alpha \lambda_{2}\right)}{|a|}$, se llega a $\operatorname{Re}\left(\alpha \lambda_{2}\right)+|a| r^{m-1}<0$ y por tanto

$$
\operatorname{Re}\left(\left\langle\alpha \varphi_{z_{0}}^{\prime}(0), z_{0}\right\rangle\right)<0
$$

Se sigue que para $r>0$ suficientemente pequeño, $L$ intersecta transversalmente a cada una de las esferas $S_{r}^{3}$. De ésta manera $L \cap S_{r}^{3}$ es una curva real. Observe que si $L=\mathbb{C}^{*} \times\{(0,0)\}$ entonces ésta curva es el círculo $S_{r}^{1} \times\{0\}$. Analicemos esta intersección para $L \in \mathcal{F}_{Z}-\left\{\left\{(0,0), \mathbb{C}^{*} \times\{(0,0)\}\right\}\right.$.

Sea $\left(T_{k}\right) \subseteq \mathbb{C}$ tal que $\lim _{k \rightarrow \infty}\left|T_{k}\right|=+\infty$ y $\varphi_{z_{0}}\left(T_{k}\right) \in L \cap S_{r}^{3}$, recordemos que $\varphi_{z_{0}}: \mathbb{C} \rightarrow \mathbb{C}^{2}$ viene dado por

$$
\varphi_{z_{0}}(T)=\left(\mu_{1}(T), \mu_{2}(T)\right)=\left(\left(z_{1}+a z_{2}^{m} T\right) e^{m \lambda_{2} T}, z_{2} e^{\lambda_{2} T}\right)
$$

Observe que

$$
\frac{\left|\mu_{1}(T)\right|}{\left|\mu_{2}(T)\right|^{m}}=\frac{\left|z_{1}+a z_{2}^{m} T\right| \cdot\left|e^{m \lambda_{2} T}\right|}{\left|z_{2}\right|^{m} \cdot\left|e^{\lambda_{2} T}\right|^{m}}=\frac{\left|z_{1}+a z_{2}^{m} T\right|}{\left|z_{2}\right|^{m}}, \quad \forall T \in \mathbb{C}
$$

luego

$$
\lim _{k \rightarrow \infty} \frac{\left|\mu_{1}\left(T_{k}\right)\right|}{\left|\mu_{2}\left(T_{k}\right)\right|^{m}}=\lim _{k \rightarrow \infty} \frac{\left|z_{1}+a z_{2}^{m} T_{k}\right|}{\left|z_{2}\right|^{m}}=+\infty
$$

Por otro lado como $\left|\mu_{1}\left(T_{k}\right)\right|^{2}+\left|\mu_{2}\left(T_{k}\right)\right|^{2}=r^{2}, \forall k \in \mathbb{N}$, tenemos que $\left|\mu_{1}\left(T_{k}\right)\right|=\sqrt{r^{2}-\left|\mu_{2}\left(T_{k}\right)\right|^{2}}$ y por tanto

$$
\frac{\left|\mu_{1}\left(T_{k}\right)\right|}{\left|\mu_{2}\left(T_{k}\right)\right|^{m}}=\sqrt{\frac{r^{2}-\left|\mu_{2}\left(T_{k}\right)\right|^{2}}{\left|\mu_{2}\left(T_{k}\right)\right|^{m}}}, \quad \forall k \in \mathbb{N}
$$

luego

$$
\lim _{k \rightarrow \infty} \frac{r^{2}-\left|\mu_{2}\left(T_{k}\right)\right|^{2}}{\left|\mu_{2}\left(T_{k}\right)\right|^{2 m}}=+\infty
$$


concluimos que $\lim _{k \rightarrow \infty}\left|\mu_{2}\left(T_{k}\right)\right|=0$. Un resultado análogo se consigue cuando tomamos $\left(T_{j}\right) \subseteq \mathbb{C}$ tal que $\lim _{j \rightarrow \infty}\left|T_{j}\right|=0$ y $\varphi_{z_{0}}\left(T_{j}\right) \in L \cap S_{r}^{3}$.

Concluimos que de las curvas que provienen al intersectar las hojas de $\mathcal{F}_{Z}-\{(0,0)\}$ con la esfera $S_{r}^{3}$ (para $r>0$ suficientemente pequeño), sólo una de ellas es cerrada, las demás son abiertas y se enrollan en ambos sentidos a la anterior.

\section{REFERENCIAS BIBLIOGRÁFICAS}

[1] R. Benazic, Tópicos de dinámica compleja, Notas de clase, Lima (2008).

[2] R. Benazic, Singularidades de Campos Vectoriales Holomorfos en el Dominio de Poincaré, PRO MATHEMATICA, Vol. X Nos. 19-20 (1996), pp. 9 - 33.

[3] R. Gunning - H. Rossi, Analytic Functions of Several Complex Variables, Prentice-Hall (1965).

[4] E. Hille, Ordinary differential equations in the complex domain, Dover, (1976).

[5] J. Palis - W. de Mello, Introdução a os sistemas dinâmicos,Projeto Euclides, IMPA (1978).

[6] J. Sotomayor, Lições de Equações Diferenciais Ordinárias, Projeto Euclides, IMPA (1979). 